\title{
Research on Ethernet Communication and Remote Control of PLC and $\mathrm{PC}$ \\ Li Ying $^{1}$, Li Yao ${ }^{2}$
}

(School of Mechanical \& Power Engineer, East China University of Science

And Technology, Shanghai, 200237, China)

Keywords: LabVIEW; Modbus TCP/IP; Ethernet; Communication; Web Server; PLC

\begin{abstract}
The communication mode of PC and PLC based on Web and Ethernet is made of PC, PLC and Ethernet module .In the view of the limitation of the scope of PLC control, the poor integration of control system, the bad unified control of real-time, and the communication mode of PC and PLC based on Web and Ethernet is proposed. A hardware system which included LabVIEW web server, PLC, Ethernet module, TP-Link, and stepper motors is designed. In addition, the software system of the Ethernet communication between PC and PLC based on TCP/IP Modbus protocol is developed by using the LabVIEW graphics software. Through the Web server of LabVIEW, the VI control panel is released to the Internet aiming at achieving the remote control. The feasibility of this communication mode is verified by the experiment of the speed feedback of the encoder.
\end{abstract}

\section{Introduction}

In the field of industrial control, PLC is applied widely in actual production because of high reliability, strong anti-interference ability, flexible function module, easy combination, but because there are all kinds of the Field buses, making the integration of control system inconvenience. In addition, the current control of the PLC is limited to the close range of control, but also to limit the construction of a fully open, fully decentralized, unified control of industrial control system [1]. Therefore, on account of the TCP / IP protocol, the LabVIEW of TCP / IP function and LabVIEW integrated remote panel technology, the article put forward the communication mode of PC and PLC based on Web and Ethernet by using PLC Ethernet module.

\section{The component of the communication system between PC and PLC based on Web and Ethernet}

Hardware system and software system comprise the communication system between PC and PLC based on Web and Ethernet.

\section{The component of hardware system}

Figure 1 shows the system hardware components, it consists of the client PC, LabVIEW server, TP-Link, PLC, stepper motor group. The remote client get access to the Internet by visiting VI Web server based on LabVIEW remote panel technology [2]. Remote clients can achieve remote control by control panel without installing LabVIEW, DCI Soft and WPL Soft software, so the remote control mode makes those nonprofessional technical personnel easy to operation in the control process. In addition, it has achieved the goal of remote control, real-time data acquisition and processing. LabVIEW Web PC server connect to PLC Ethernet module of the static IP through the TP-Link LAN .Data transmission is based on the TCP/IP Modbus protocol to achieve the function of the instruction code (data frame) transmission. 


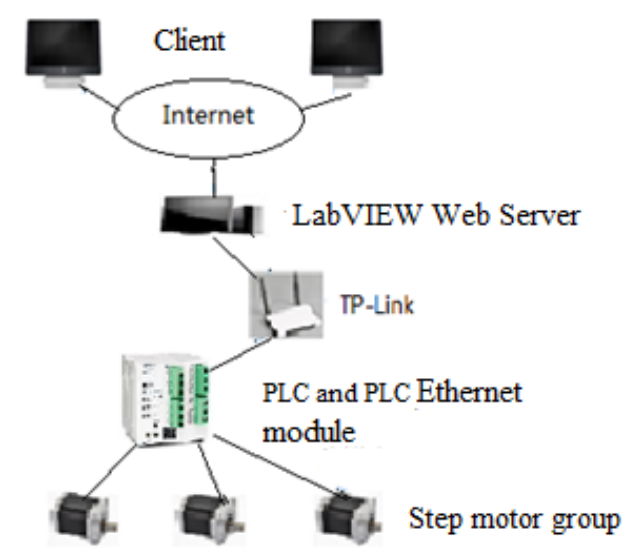

Fig.1 Hardware System Composition

\section{The component of software system}

The software system of the communication between PC and PLC based on Web and Ethernet consists of PC and PLC, including the PC cover remote clients have access to visiting to the LabVIEW of Web server and the Ethernet communication between PC and PLC, while PLC includes the Ethernet communication of between PLC and PC. Communication protocol is Modbus TCP / IP protocol and Ethernet communication realized by PLC module, the component of software system as shown in Figure 2.

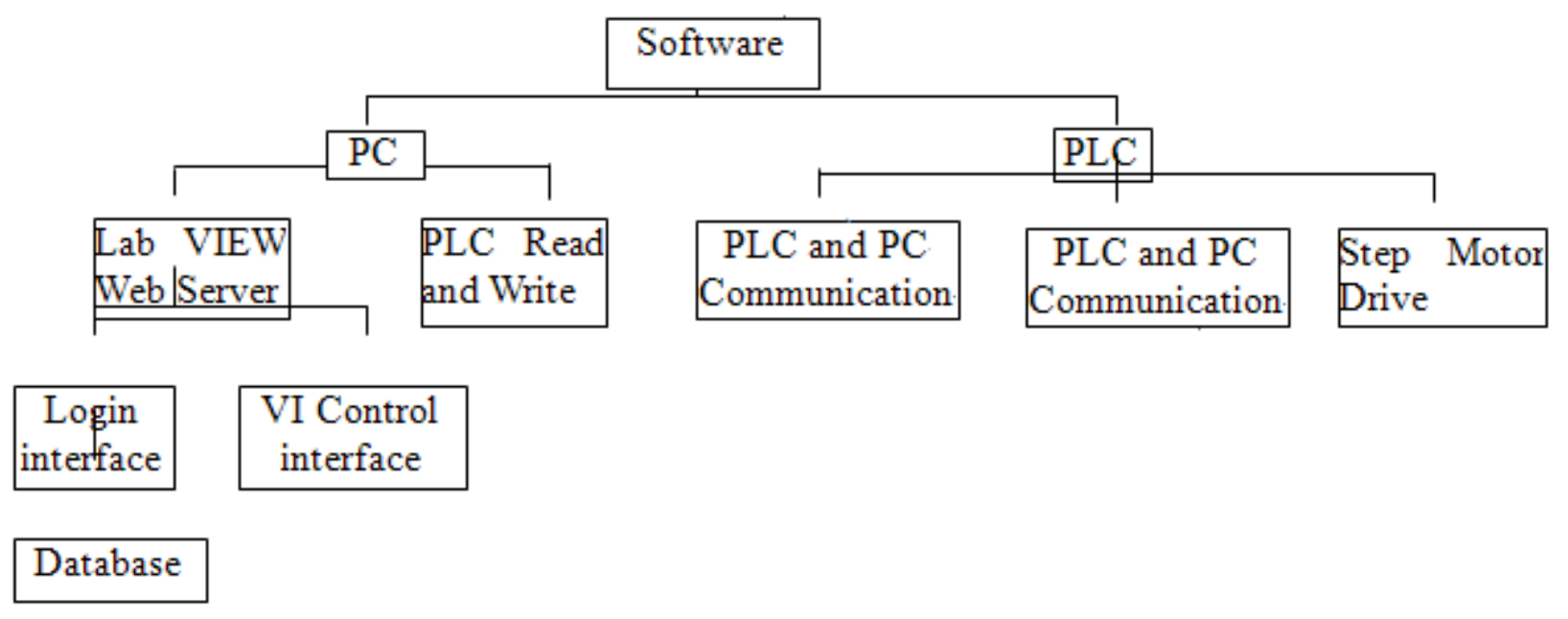

Fig.2 Software System Composition

\section{Communication protocol and Communication process}

\section{Modbus TCP/IP protocol}

The communication protocol of the communication mode of PC and PLC based on Web and Ethernet is Modbus TCP/IP protocol, TCP/IP Modbus protocol is formed on the Modbus application layer protocol, and its data (ADU) contains the message header, function code, data 3 parts. As shown in table 1.

Table1 Modbus TCP/IP data frame

\begin{tabular}{c|c|c}
\hline MBAP header & Function code & data \\
\hline
\end{tabular}

The length of MBAP Header is seven byte, it is composed of four parts [3] such as the transaction identifier for the transmission symbol, in master / slave communication the sign of MODBUS request or response of transmission, usually by the master to generate, from the station in response to a request for copy the value; protocol identifier for the deal marks, its value is 0 that indicates the Modbus protocol, while the value is 1which indicates UN-TE protocol; Length 
indicates the length of the sum of the domain subsequent bytes; Unit identifier is mark unit, used to define the device address of the station.

Table.2 MBAP Header

\begin{tabular}{l|c|l|l}
\hline Transaction & Protocol Identified & Length & Unit Identifier \\
\hline 0000 & 0000 & 0006 & 01 \\
\hline
\end{tabular}

MBAP header the transaction identifier usually sets to 0000 , indicates the server copy; protocol identifier usually sets to 0000 , indicates MODBUS protocol; the value of Length is 0600 , representing the data length; unit identifier value is 01 indicates the device address of the station.

\section{Communication process}

According to communication mode, remote control system is divided into two types: client and server mode (C/S mode) and browser / server mode (B/S mode) [4]. B/S simplifies the client, you do not have to install different programs in different clients, and can also set the permissions of access to improve the security of the system. The system update is mainly completed by the Web server. LabVIEW in the remote panel (Front Panel Remote) technology is one of B/S mode [5], the customers have access to acquiring the power of control mainly through the Web LabVIEW server.

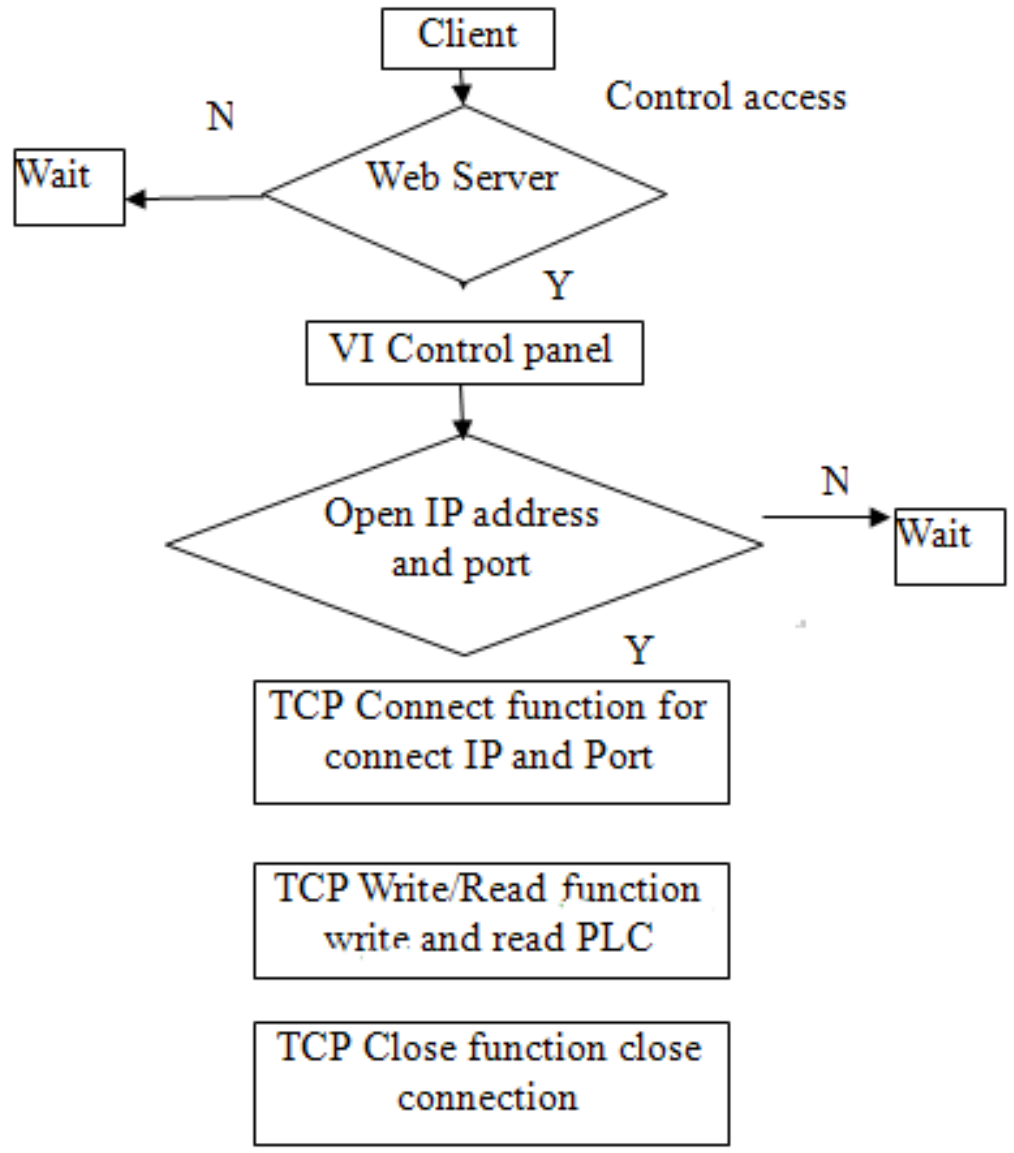

Fig.3 Communication Flowchart

As shown in Figure 3, the clients get control permissions from the Web LabVIEW server for controlling the VI front panel. When PLC Ethernet ports open, LabVIEW adopts embedded TCP / IP protocol of network communication, this paper makes PLC as the server and PC as the client, using LabVIEW node function TCP Open [6], the port number 502 and the default IP of DVPEN01-SL to establish the connection between PC and PLC. For example, IP's DVPEN01-SL address: [192.168.0.4]; subnet mask: [255.255.255.0]; default gateway: PC; IP [192.168.0.1] for 192.168.0.3. Use the write function of TCP nodes to send function instruction code to PLC, for example, a group of data frames for 0000000000060105 0800FF00, 000000000006 represents fixed data packet headers, 01 indicates the PLC of station number, 05 represents the function code of drive a single coil, 0800FF00 indicates stepper motor driver data code expression. 


\section{Communication and control experiment}

In order to verify the feasibility of the communication between PLC and PC based on Web and Ethernet, That the PC read and write operation of PLC is carried out under the condition of Ethernet communication in Ethernet module. In the experiment, it is mainly to monitor and adjust the speed of the 57BYGH250C step motor, the upper computer is based on the LabVIEW development of the man-machine interface and monitoring interface. In communications, the LabVIEW of TCP toolkit and DVPEN01 Ethernet module finish communication connection in TP-Link Lan. Information feedback, the rotary encoder stepper motor speed real-time feedback to the PLC, and then, the host computer read or write the PLC of internal register, thus completing the data interaction of the host computer and stepper motor.

Figure 4 shows that PC and PLC achieve Ethernet communication through the PLC of IP address 192.168.0.4 and port number 502. Using TCP/IP Modbus communication protocol, the header part of the code is 00000000000601 , the function code is 03, the data is 10000001 . This data frame convey the command of read the internal register D0 to PLC, read TCP function starts reading the encoder registers in the register D0, the sampling period of the program is 1 second. Finally the LabVIEW graphical interface display the stepper motor speed feedback.

Fig.4 Stepper Motor Encoder Feedback Code

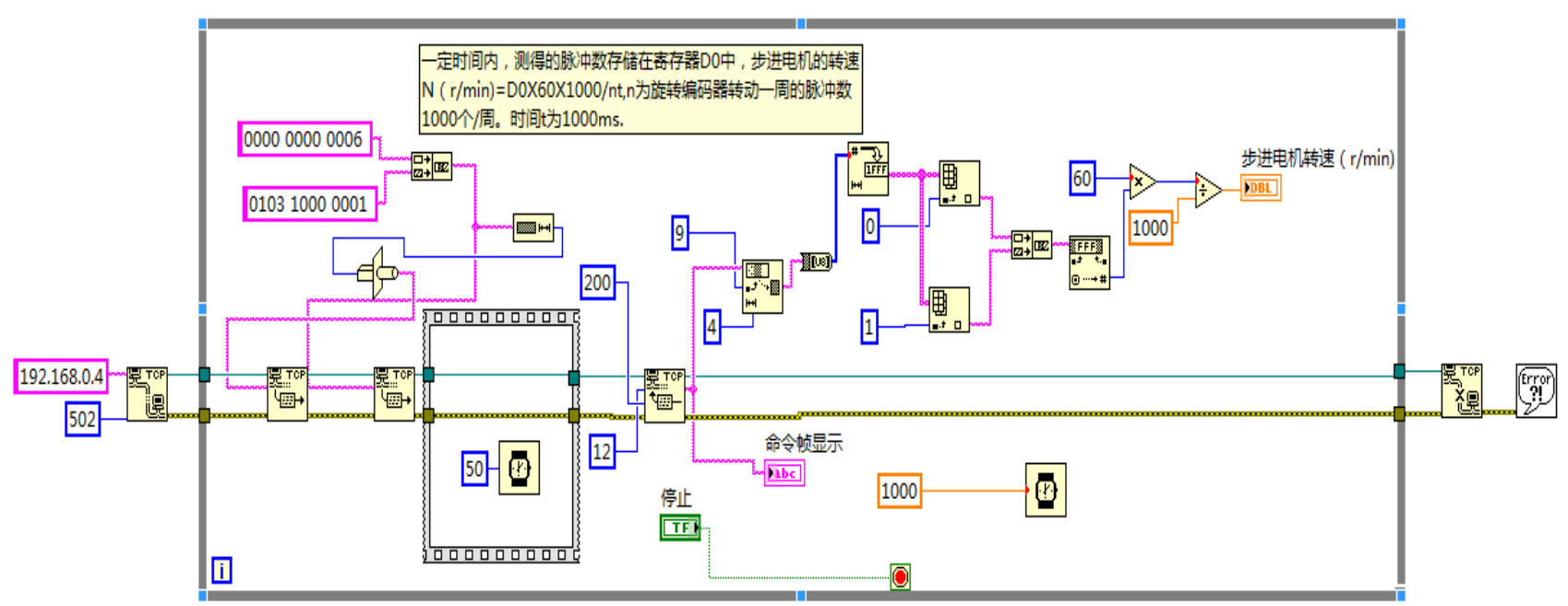

In addition, written in WPLSOFT PLC program code [8] as shown in Figure 5, which PLSY k1000 K0 Y0 said sends pulse frequency $1000 \mathrm{~Hz}$ to PLC continuously, pulse infinite number of pulses, SPD x1 k1000 d0 represented read send existing registers d0 pulse number every second , the formula $\mathrm{n}=60 *[10] \wedge 3 \mathrm{x}$ D0/nt ( $\mathrm{n}$ for the encoder to turn a circle of pulse number is 1000 , and $\mathrm{t}$ is the pulse time $1000 \mathrm{~ms}$ ) is used for translating into stepper motor speed.

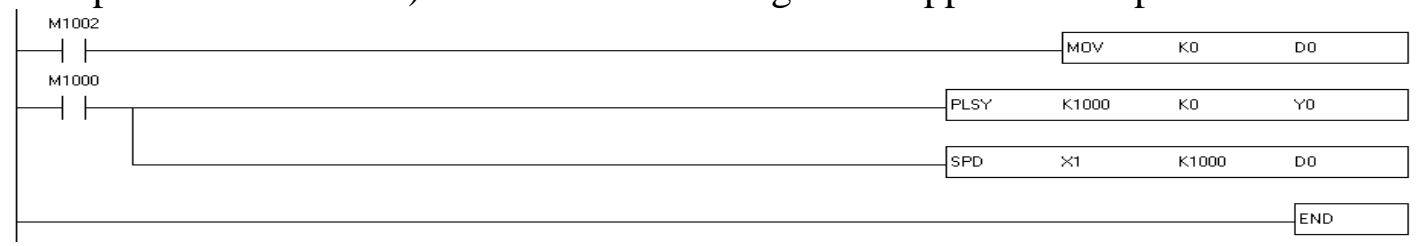

Fig.5 PLC Program Executing Code

Due to the LabVIEW embedded web server function, so there is no need to develop a web service application, only need simple to set up remote clients can upgrade the original stand-alone version of the measurement and control system for web remote measurement and control system. Remote clients can access the web publishing LabVIEW VI in VI control conditions to achieve remote control client, according to the need of actual production, the user can set the parameter values in order to achieve the control objective, the remote control interface as shown in Figure 6. 


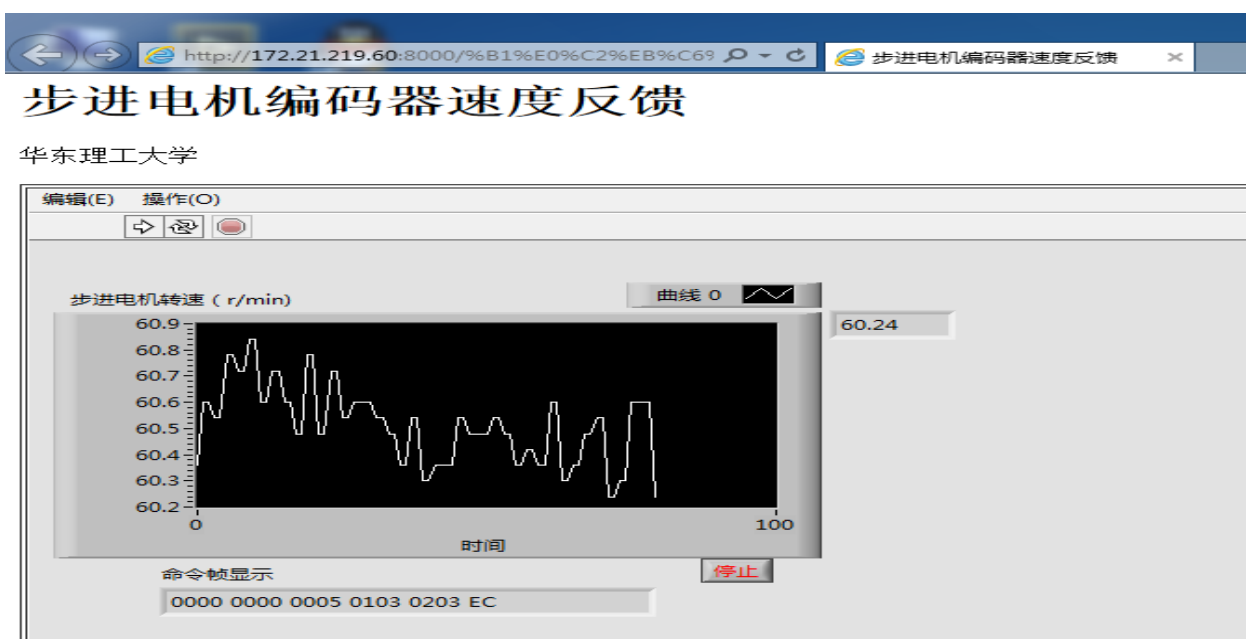

Fig.6 Remote Control Interface

\section{Conclusion}

The control mode of Web page has changed the traditional one to one, short distance control mode, this kind of control mode is easy to operate for nonprofessionals, and strong real-time operation enable the operator conduct real-time data interaction [9] with the control object. LabVIEW graphics software remote panel technology enables Web server easy to create and accessible. In addition, in the communication between PC and PLC. Compared with the traditional RS232 serial communication, Ethernet communication mode not only in the data transmission speed and the amount of data occupy obvious advantages, importantly, it expands the communication range and the lower position machine control, not just a PC controls a PLC, but a PC can conduct with a plurality of PLC to realize the data read and write between PLC and can be used for data of each transmission, greatly increased the flexibility of real-time control, speed, distance [10].

\section{References}

[1] Shi Su-min, Liu Jian-xin, Jin-Peng, Wu Qin-bin. Development of Ethernet Communication System between PLC and PC for Automobile Sunroof Testing Control System [J].Machine Tool\&Hydraulics.2013, 41(16):132-134

[2] Li Ji-rong , He Xiang-chu .Two Methods to Publish Web Page by LabVIEW Wed Sever[J].Instrumentation Technology,2003,(5):13-15

[3] Zhou Wen-yi, Fang Jun-ya, Zhu Zi-huan. Control System of Servo Motor Based on LabVIEW and Modbus/TCP[J].Measurement \& Control Technology.2015,34(3):83-85

[4] Zhao Xin-hua..Remote Virtual Instrument technology Based on LabVIEW[D].Harbin: School of Mechanical and Engineering ,Harbin University of Science and Technology,2005) [5]Liu Chuan-hui, Liu Ji-sheng. Research on application of remote-control of machine using VI[J].Machinery Design \&Manufacture.2007,(2):90-91

[6] Chen Xi-hui,Zhang Yin-hong. LabVIEW8.20 Programming From entry to the master [M].Beijing: Tsinghua University Publishing House,2007..

[7] DVP0204310-02,DVPEN01-SL.Ethernet Communication Module Operation Manual[S].2012

[8] Zhang Xi-chuan. Application of delta ES/EX/SS series PLC technology [M].Beijing:China Power Press,2009

[9] Xiong Wei-li, Tang Bin-bin, Chen Min-fang. Online Remote water Treatment Monitoring System Based on LabVIEW and Web Technologies [J].Process Automation 
Instrumentation,2012,33(8);41-44.

[10] Liang De-chun, Fang Jiang-long, Tao Yi-min . Design of Ethernet Communication of NC System[J].Machinery, 2008,46(1):33-35 